\title{
Further Results on Stability Analysis for Markovian Jump Systems with Time-Varying Delays
}

\author{
O. M. Kwon, ${ }^{1}$ R. Sakthivel, ${ }^{2,3}$ and M. J. Park ${ }^{1}$ \\ ${ }^{1}$ School of Electrical Engineering, Chungbuk National University, 1 Chungdae-ro, Seowon-gu, Cheongju 362-763, Republic of Korea \\ ${ }^{2}$ Department of Mathematics, Sri Ramakrishna Institute of Technology, Coimbatore, Tamilnadu 641010, India \\ ${ }^{3}$ Department of Mathematics, Sungkyunkwan University, Suwon 440-746, Republic of Korea
}

Correspondence should be addressed to O. M. Kwon; madwind@chungbuk.ac.kr and R. Sakthivel; krsakthivel@yahoo.com

Received 15 May 2015; Revised 1 July 2015; Accepted 1 July 2015

Academic Editor: Leonid Shaikhet

Copyright (C) 2015 O. M. Kwon et al. This is an open access article distributed under the Creative Commons Attribution License, which permits unrestricted use, distribution, and reproduction in any medium, provided the original work is properly cited.

\begin{abstract}
This paper is concerned with the problem of stability analysis for Markovian jump systems with time-varying delays. By constructing a newly augmented Lyapunov-Krasovskii functional and combining Wirtinger-based integral inequality, an improved delaydependent stability criterion within the framework of linear matrix inequalities (LMIs) is introduced. Based on the result of delaydependent stability criterion, when linear systems have fast time-varying delays, a corresponding stability condition is given. Via three numerical examples, the improvements of the proposed criteria are shown by comparing maximum delay bounds provided by our theorems with the recent results.
\end{abstract}

\section{Introduction}

Stability analysis of dynamic systems is a prerequisite and essential job before designing a controller to achieve the prescribed specifications. In particular, a great concern of stability analysis for systems with time-delays has been received due to the fact that time-delay naturally occurs in many practical systems such as networked control system, chemical processing, hot rolling mill, synchronization between chaotic systems, neural networks, and multiagent systems. For instance, see $[1,2]$ and references therein.

The main issue in delay-dependent stability analysis for time-delay systems with the framework of LMIs is how to increase maximum delay bounds for guaranteeing the asymptotic stability of systems. Thus, the choosing of Lyapunov-Krasovskii functional (LKF) and some techniques in estimating an upper bound of time-derivative value of the constructed LKF are the most important factors in enhancing the stability feasible region. In the LKF aspect, quadratic form, single integral, and double integral of quadratic form are the most utilized functionals. Recently, since the triple integral form of LKF was introduced in [3], this form of LKF has been utilized in many works such as [4-6]. Moreover, in
$[4,5]$, it was shown that some augmented LKFs can increase the feasible region of stability criteria. In estimating an upper bound of time-derivative value of LKF, Jensen's inequality [7], free-weighting matrix technique [8], and reciprocally convex optimization theory [9] make big impacts on the enhancement of delay-dependent stability and stabilization. Seuret and Gouaisbaut [10] proposed the Wirtinger-based integral inequality which provides more tight lower bounds than Jensen's inequality and showed that the utilization of Wiritinger-based integral inequality can improve maximum delay bounds in many systems such as systems with constant and known delay, systems with a time-varying delay, systems with a constant distributed delay, and sampled-date systems. Cheng and Xiong [11] reduced conservative condition of stabilization criteria for continuous-time systems with timevarying input by introducing a new integral inequality. Recently, in $[12,13]$, for neural network with time-varying delay, it can be confirmed that the utilization of Wirtingerbased integral inequality in obtaining an upper bound of time-derivative values of some augmented LKFs can provide larger delay bounds than some other literatures. Very recently, in [14], it was shown that the results obtained by [10] can be further improved by choosing some new augmented 
LKFs. From the statements mentioned above, one can see that the choosing of LKF and some techniques play key roles to reduce the conservatism of stability criteria.

On the other hand, increasing attention has been paid to Markovian jumping systems (MJSs) which are a special sort of hybrid systems and driven by Markov chain. MJSs may undergo unexpected changes in their structure and parameters including economic systems, aerospace systems, power systems, and networked control system $[15,16]$. Very recently, a survey on recent developments of modeling, analysis, and design of MJSs was reported in Shi and Li [17].

In this regard, many researchers put their times and efforts into stability and stabilization of Markovian jumping systems with time-delays. In [18], the problems of robust $\mathscr{H}_{\infty}$ control and $\mathscr{H}_{\infty}$ filtering for uncertain MJSs with time-varying delays were investigated by utilizing bounded real lemma. In [19], some new results on stabilization of MJSs with time-delays were proposed based on a delaypartitioning approach. Wu et al. [20] investigated the problem of stability and $\mathscr{H}_{\infty}$ filtering for singular Markovian jump systems with time-delay via a delay-dependent bounded real lemma. Li et al. [21] utilized an input-output approach to stability and stabilization of MJSs with time-varying delays and showed the reduction of conservatism of the concerned criteria by a precise approximation of time-varying delay. By constructing new LKFs having distinct Lyapunov matrices for different modes, the mean square exponential stability and stabilization problems were studied in [22] for MJSs with constant time-delays. In [23], improved delay-dependent stability and $\mathscr{H}_{\infty}$ control for singular Markovian jump systems with time-delay by utilizing delay-partitioning technique with a tuning parameter. Zhu [24] derived some new conditions for ensuring the asymptotic stability of singular nonlinear MJSs with unknown parameters and continuously distributed delays. Recently, some new augmented LKFs and techniques in estimating upper bounds of time-derivative of LKFs were introduced in [25] in studying stability and $\mathscr{H}_{\infty}$ performance analysis of MJSs with time-varying delays. Very recently, in [26], an input-output approach to the delaydependent stability analysis and $\mathscr{H}_{\infty}$ control for MJSs with time-varying delays and deficient transition descriptions. The problem of finite-time $\mathscr{H}_{\infty}$ estimation for a class of discretetime Markov jump systems with time-varying transition probabilities subject to average dwell time switching was investigated in [27]. However, as mentioned in [17], the results on stability have still some conservativeness. Thus, there are rooms for further reduction of conservativeness caused by time-delays with the construction of a newly augmented Lyapunov-Krasovskii functional and utilization of a Wirtinger-based integral inequality [10].

Motivated by [17] and based on the result of [25], the goal of this paper is to propose a further improved result of delay-dependent stability for MJSs with time-varying delays. In Theorem 5, a new and improved stability criterion will be proposed based on the results of [25]. To derive less conservative results, Wirtinger-based integral inequality is applied to the augmented LKFs and some new techniques are introduced. When an upper bound of time-derivative value of time-varying delay is larger than one or unknown, a corresponding result will be presented in Corollary 6 by constructing some part of LKF utilized in Theorem 5. Comparing with the result of [25], the constructed Lyapunov-Krasovskii functionals in Theorem 5 and Corollary 6 are simple since the triple and quadruple integral form of Lyapunov-Krasovskii functionals will not be utilized. Via three numerical examples, the advantage and effectiveness of the proposed results will be explained by comparing maximum delay bounds with some recent results presented in other literatures.

Notation. Throughout this paper, the following notations will be used. $X>0(X \geq 0)$ means that $X$ is a real symmetric positive definitive matrix (positive semidefinite). The subscript " $T$ " represents the transpose. $X^{\perp}$ denotes a basis for the null-space of $X . \mathbb{R}^{n}$ denotes the $n$-dimensional Euclidean space and $\mathbb{R}^{m \times n}$ is the set of all $m \times n$ real matrix. $\mathscr{C}_{n, h}=\mathscr{C}\left([-h, 0], \mathbb{R}^{n}\right)$ denotes the Banach space of continuous functions mapping the interval $[-h, 0]$ into $\mathbb{R}^{n}$ with the topology of uniform convergence. $\mathscr{L}_{2}[0, \infty)$ means the space of square-integrable vector functions over $[0, \infty)$. $\mathscr{E}\{\cdot\}$ denotes the expectation operator with respect to some measure $\mathscr{P} . I_{n}, 0_{n}$, and $0_{m \cdot n}$ denote $n \times n$ identity matrix and $n \times n$ and $m \times n$ zero matrices, respectively. $\|\cdot\|$ refers to the induced matrix 2-norm. $\operatorname{diag}\{\ldots\}$ denotes the block diagonal matrix. $X_{[f(t)]} \in \mathbb{R}^{m \times n}$ means that the elements of matrix $X_{[f(t)]}$ include the scalar value of $f(t)$. For any matrix $M, \operatorname{Sym}\{M\}$ means $M+M^{T} \cdot \operatorname{col}\left\{x_{1}, x_{2}, \ldots, x_{n}\right\}$ means $\left[x_{1}^{T}, x_{2}^{T}, \ldots, x_{n}^{T}\right]^{T}$.

\section{Problem Statement and Preliminaries}

Consider the Markovian jump system with time-varying delays:

$$
\begin{aligned}
& \dot{x}(t)=A(r(t)) x(t)+A_{d}(r(t)) x(t-h(t)), \\
& x(t)=\phi(t), \quad \forall t \in\left[-h_{U}, 0\right],
\end{aligned}
$$

where $x(t) \in \mathbb{R}^{n}$ is the state vector, $\phi(t)$ which belongs to $\mathscr{C}_{n, h_{U}}$ means the initial function, $A(r(t))$ and $A_{d}(r(t))$ are known system matrices with appropriate dimensions, and $r(t)$ denotes a finite state Markovian jump process representing the system mode. That is, $r(t)$ takes values in the finite discrete set $\mathcal{S}=\{1,2, \ldots, N\}$ with transition probability matrix $\Pi=\left[\pi_{i j}\right]$.

The transition probability is described as

$$
\begin{aligned}
\operatorname{Pr}\{r(t+\delta)=j \mid r(t)=i\} & \\
& = \begin{cases}\pi_{i j} \delta+o(\delta), & j \neq i, \\
1+\pi_{i j} \delta+o(\delta), & j=i,\end{cases}
\end{aligned}
$$

where $\delta>0, \lim _{\delta \rightarrow 0^{+}(o(\delta) / \delta)=0}, \pi_{i j} \geq 0$ for $j \neq i$ and $\pi_{i i}=$ $-\sum_{j \neq i} \pi_{i j}$.

The delay in states, $h(t)$, is a time-varying and continuous function satisfying

$$
\begin{aligned}
0 & \leq h(t) \leq h_{U}, \\
h(t) & \leq h_{D},
\end{aligned}
$$


where $h_{U}$ is a known positive scalar and $h_{D}$ is any constant one.

For simplicity, a matrix $M(r(t))$ of $i$ th node is denoted by $M_{i}$ for each possible $r(t)=i, i \in \mathcal{S}$ in the rest of this paper. For example, $A(r(t))$ and $A_{d}(r(t))$ of $i$ th node will be represented as $A_{i}$ and $A_{d i}$, respectively. Let $x_{t}=x(t+s)$ for $s \in\left[-h_{U}, 0\right]$. From [28], it should be noted that $\left\{\left(x_{t}, r(t)\right)\right\}$ is a Markov process for $t \geq 0$. Then, its weak infinitesimal operator $\mathscr{L}$ acting on a functional $V\left(x_{t}, i\right)$ is defined by

$$
\begin{aligned}
\mathscr{L} V & \left(x_{t}, i\right) \\
& =\lim _{\delta \rightarrow 0^{+}} \frac{1}{\delta}\left[\mathscr{E}\left\{V\left(x_{t+\delta}, r(t+\delta) \mid x_{t}, r(t)=i\right)\right\}\right. \\
& \left.-V\left(x_{t}, i\right)\right] .
\end{aligned}
$$

In stability analysis of system (1), the following definition will be utilized.

Definition 1 (see [29]). For any finite $\phi(t) \in \mathscr{C}_{n, h_{U}}$, and the initial condition of the mode $r_{0} \in \mathcal{S}$, the system $\dot{x}(t)=$ $A(r(t)) x(t)+A_{d}(r(t)) x(t-h(t))$ is said to

(a) be stochastically stable if there exists a constant $T\left(r_{0}, \phi(t)\right)$ such that

$$
\mathscr{E}\left\{\int_{0}^{\infty}\|x(t)\|^{2} \mid r_{0}, \phi(t)\right\} \leq T\left(r_{0}, \phi(t)\right),
$$

(b) be mean square stable if

$$
\lim _{t \rightarrow \infty} \mathscr{E}\|x(t)\|^{2}=0
$$

hold for any initial condition $\left(r_{0}, \phi(t)\right)$,

(c) be mean exponentially stable if there exist constants $\alpha>0$ and $\beta>0$ such that the following holds for any initial condition $\left(r_{0}, \phi(t)\right)$ :

$$
\mathscr{E}\left\{\|x(t)\|^{2} \mid r_{0}, \phi(t)\right\} \leq \alpha\|\phi(t)\| e^{-\beta t} .
$$

Based on the results of [25], the objective of this paper is to develop further improved delay-dependent stability criteria of system (1) which will be conducted in next section.

The following lemmas will be utilized in deriving main results.

Lemma 2. Consider a given matrix $M>0$. Then, for all continuous function $\eta$ in $[a, b] \rightarrow \mathbb{R}^{n}$, the following inequality holds:

$$
\begin{aligned}
& \int_{a}^{b} \eta^{T}(s) M \eta(s) d s \geq \frac{1}{b-a}\left(\int_{a}^{b} \eta(s) d s\right)^{T} \\
& \cdot M\left(\int_{a}^{b} \eta(s) d s\right) \\
& +\frac{3}{b-a}\left(\int_{a}^{b} \eta(s) d s-\frac{2}{b-a} \int_{a}^{b} \int_{s}^{b} \eta(u) d u d s\right)^{T} \\
& \cdot M\left(\int_{a}^{b} \eta(s) d s-\frac{2}{b-a} \int_{a}^{b} \int_{s}^{b} \eta(u) d u d s\right) .
\end{aligned}
$$

Proof. From the original Wirtinger-based integral inequality [10], since

$$
\begin{aligned}
\int_{a}^{b} \eta(s) d s-\frac{2}{b-a} \int_{a}^{b} \int_{a}^{s} \eta(u) d u d s \\
=\int_{a}^{b} \eta(s) d s \\
\quad-\frac{2}{b-a} \int_{a}^{b}\left(\int_{a}^{b} \eta(u) d u-\int_{s}^{b} \eta(u) d u\right) d s \\
=\int_{a}^{b} \eta(s) d s-\frac{2}{b-a} \underbrace{b}_{a} \int_{a}^{b} \eta(u) d u d s \\
\quad+\frac{2}{b-a} \int_{a}^{b} \int_{s}^{b} \eta(u) d u d s \\
=-\int_{a}^{b} \eta(s) d s+\frac{2}{b-a} \int_{a}^{b} \int_{s}^{b} \eta(u) d u d s,
\end{aligned}
$$

inequality (8) holds.

Lemma 3 (see [30]). Let $\zeta \in \mathbb{R}^{n}, \Phi=\Phi^{T} \in \mathbb{R}^{n \times n}$, and $B \in \mathbb{R}^{m \times n}$ such that $\operatorname{rank}(B)<n$. Then, the following two statements are equivalent:

(a) $\zeta^{T} \Phi \zeta<0, B \zeta=0, \zeta \neq 0$,

(b) $\left(B^{\perp}\right)^{T} \Phi B^{\perp}<0$, where $B^{\perp}$ is a right orthogonal complement of $B$.

Lemma 4 (see [31]). For the symmetric appropriately dimensional matrices $\Omega>0, \Xi$, an any matrix $\Lambda$, the following two statements are equivalent:

(a) $\Xi-\Lambda^{T} \Omega \Lambda<0$,

(b) there exists a matrix of appropriate dimension $\Psi$ such that

$$
\left[\begin{array}{cc}
\Xi+\Lambda^{T} \Psi+\Psi^{T} \Lambda & \Psi^{T} \\
\Psi & -\Omega
\end{array}\right]<0 .
$$

\section{Main Results}

In this section, improved delay-dependent stability criteria for MJSs (1) will be proposed. To express vectors and matrices in simple forms, block entry matrices $e_{i}(i=1, \ldots, 9) \in \mathbb{R}^{9 n \times n}$ will be used. For example, $e_{3}$ means $\left[0_{n \cdot 2 n}, I_{n}, 0_{n \cdot 6 n}\right]^{T}$. And some of scalars, vectors, and matrices are defined as

$$
\begin{gathered}
\zeta(t)=\operatorname{col}\left\{x(t), x(t-h(t)), x\left(t-h_{U}\right), \dot{x}(t),\right. \\
\dot{x}\left(t-h_{U}\right), \int_{t-h(t)}^{t} x(s) d s, \int_{t-h_{U}}^{t-h(t)} x(s) d s,
\end{gathered}
$$




$$
\begin{aligned}
& \left.\int_{t-h(t)}^{t} \int_{s}^{t} x(u) d u d s, \int_{t-h_{U}}^{t-h(t)} \int_{s}^{t} x(u) d u d s\right\} \\
& \eta(t)=\operatorname{col}\left\{x(t), x\left(t-h_{U}\right), \int_{t-h_{U}}^{t} x(s) d s,\right. \\
& \left.\int_{t-h_{U}}^{t} \int_{s}^{t} x(u) d u d s\right\} \\
& \alpha(t, s)=\operatorname{col}\left\{\dot{x}(s), x(s), \int_{s}^{t} \dot{x}(u) d u\right\}, \\
& \beta(t, s)=\operatorname{col}\left\{x(s), \int_{s}^{t} \dot{x}(u) d u\right\} \text {, } \\
& Q_{\text {aug } 1}=\mathscr{Q}+\left[\begin{array}{lll}
0_{n} & P_{1} & 0_{n} \\
P_{1} & 0_{n} & 0_{n} \\
0_{n} & 0_{n} & 0_{n}
\end{array}\right] \text {, } \\
& Q_{\text {aug2 }}=\mathscr{Q}+\left[\begin{array}{lll}
0_{n} & P_{2} & 0_{n} \\
P_{2} & 0_{n} & 0_{n} \\
0_{n} & 0_{n} & 0_{n}
\end{array}\right] \text {, } \\
& \Xi_{1 i}=\operatorname{Sym}\left\{\left[e_{1}, e_{3}, e_{6}+e_{7}, e_{8}+e_{9}\right]\right. \\
& \text { - } \left.\mathscr{R}_{i}\left[e_{4}, e_{5}, e_{1}-e_{3}, h_{U} e_{1}-e_{6}-e_{7}\right]^{T}\right\}, \\
& +\left[e_{1}, e_{3}, e_{6}+e_{7}, e_{8}+e_{9}\right]\left(\sum_{j=1}^{s} \pi_{i j} \mathscr{R}_{j}\right)\left[e_{1}, e_{3}, e_{6}+e_{7}, e_{8}\right. \\
& \left.+e_{9}\right]^{T} \\
& \Xi_{2 i}=\left[e_{4}, e_{1}, 0_{9 n \cdot n}\right] \mathscr{N}_{i}\left[e_{4}, e_{1}, 0_{9 n \cdot n}\right]^{T}-\left[e_{5}, e_{3}, e_{1}-e_{3}\right] \\
& \cdot \mathcal{N}_{i}\left[e_{5}, e_{3}, e_{1}-e_{3}\right]^{T} \\
& +\operatorname{Sym}\left\{\left[e_{1}-e_{3}, e_{6}+e_{7}, h_{U} e_{1}-e_{6}-e_{7}\right]\right. \\
& \left.\cdot \mathcal{N}_{i}\left[0_{9 n \cdot n}, 0_{9 n \cdot n}, e_{4}\right]^{T}\right\} \text {, } \\
& \Xi_{3 i[h(t)]}=\left[e_{1}, 0_{9 n \cdot n}\right] \mathscr{G}_{i}\left[e_{1}, 0_{9 n \cdot n}\right]^{T}-\left(1-h_{D}\right)\left[e_{2}, e_{1}\right. \\
& \left.-e_{2}\right] \mathscr{G}_{i}\left[e_{2}, e_{1}-e_{2}\right]^{T}+\operatorname{Sym}\left\{\left[e_{6}, h(t) e_{1}-e_{6}\right]\right. \\
& \left.\cdot \mathscr{G}_{i}\left[0_{9 n \cdot n}, e_{4}\right]^{T}\right\} \text {, } \\
& \Xi_{4}=\left[e_{4}, e_{1}, 0_{9 n \cdot n}\right]\left(h_{U}^{2} Q\right)\left[e_{4}, e_{1}, 0_{9 n \cdot n}\right]^{T}+2 h_{U}\left[h_{U} e_{1}\right. \\
& \left.-e_{6}-e_{7}, e_{8}+e_{9},\left(\frac{h_{U}^{2}}{2}\right) e_{1}-e_{8}-e_{9}\right] \mathcal{Q}\left[0_{9 n \cdot n},\right. \\
& \left.0_{9 n \cdot n}, e_{4}\right]^{T} \\
& \Xi_{5}=h_{U}\left(e_{1} P_{1} e_{1}^{T}-e_{2} P_{1} e_{2}^{T}+e_{2} P_{2} e_{2}^{T}-e_{3} P_{2} e_{3}^{T}\right), \\
& \Lambda_{1[h(t)]}=\left[e_{1}-e_{2}, e_{6}, h(t) e_{1}-e_{6}, h(t)\left(-e_{1}-e_{2}\right)\right.
\end{aligned}
$$

Now, we have the following theorem.

Theorem 5. For given scalars $h_{U}>0$ and $h_{D}$, system (1) is stochastically stable for $0 \leq h(t) \leq h_{U}$ and $\dot{h}(t) \leq h_{D}$ if there exist positive definite matrices $\mathscr{R}_{i} \in \mathbb{R}^{4 n \times 4 n}, \mathcal{N}_{i} \in \mathbb{R}^{3 n \times 3 n}, \mathscr{G}_{i} \in$ $\mathbb{R}^{2 n \times 2 n}$, and $\mathbb{Q} \in \mathbb{R}^{3 n \times 3 n}$, any matrices $\mathcal{S} \in \mathbb{R}^{6 n \times 6 n}$ and $\Psi \in$ $\mathbb{R}^{12 n \times 8 n}$, and any symmetric matrices $P_{1} \in \mathbb{R}^{n \times n}$ and $P_{2} \in \mathbb{R}^{n \times n}$ satisfying the following LMIs for all $r(t)=i, i \in \delta$ :

$$
\left[\begin{array}{cc}
\Phi_{i, k} & \Psi^{T} \\
\Psi & -\Omega_{3 i}
\end{array}\right]<0, \quad k=1,2
$$

where $\left\{\Phi_{i, k}\right\}_{k=1}^{2}$ means the two vertices of $\Phi_{i[h(t)]}$ with the bounds of $0 \leq h(t) \leq h_{M}$. That is, $\Phi_{i, 1}=\Phi_{i[h(t)=0]}$ and $\Phi_{i, 2}=\Phi_{i\left[h(t)=h_{U}\right]}$. 
Proof. For each $r(t)=i, i \in \mathcal{S}$, let us consider the LyapunovKrasovskii functional candidate:

$$
V\left(x_{t}, i\right)=\sum_{j=1}^{4} V_{j}\left(x_{t}, i\right),
$$

where

$$
\begin{aligned}
& V_{1}\left(x_{t}, i\right)=\eta^{T}(t) \mathscr{R}_{i} \eta(t), \\
& V_{2}\left(x_{t}, i\right)=\int_{t-h_{U}}^{t} \alpha^{T}(t, s) \mathcal{N}_{i} \alpha(t, s) d s, \\
& V_{3}\left(x_{t}, i\right)=\int_{t-h(t)}^{t} \beta^{T}(t, s) \mathscr{G}_{i} \beta^{T}(t, s) d s, \\
& V_{4}\left(x_{t}, i\right)=\int_{t-h_{U}}^{t} \int_{s}^{t} \alpha^{T}(t, u) \mathcal{Q} \alpha(t, u) d u d s .
\end{aligned}
$$

From the following relationship:

$\eta(t)$

$$
=\left[\frac{x(t)}{\frac{x\left(t-h_{U}\right)}{\int_{t-h(t)}^{t} x(s) d s+\int_{t-h_{U}}^{t-h(t)} x(s) d s}}\right]
$$$$
=\left[e_{1}, e_{3}, e_{6}+e_{7}, e_{8}+e_{9}\right]^{T} \zeta(t) \text {, }
$$

$$
\dot{\eta}(t)=\left[\frac{\dot{x}(t)}{\frac{\dot{x}\left(t-h_{U}\right)}{h_{U} x(t)-x\left(t-h_{U}\right)}}\right] \underbrace{\int_{t-h_{U}}^{t} x(s) d s}_{\int_{t-h(t)}^{t} x(s) d s+\int_{t-h_{U}}^{t-h(t)} x(s) d s}]
$$

$$
=\left[e_{4}, e_{5}, e_{1}-e_{3}, h_{U} e_{1}-e_{6}-e_{7}\right]^{T} \zeta(t),
$$

$\mathscr{L} V_{1}\left(x_{t}, i\right)$ can be represented as

$$
\begin{aligned}
\mathscr{L} V_{1}\left(x_{t}, i\right)= & 2 \eta^{T}(t) \mathscr{R} \dot{\eta}(t) \\
& +\eta^{T}(t)\left(\sum_{j=1}^{s} \pi_{i j} \mathscr{R}_{j}\right) \eta(t) \\
= & \zeta^{T}(t) \Xi_{1 i} \zeta(t),
\end{aligned}
$$

where $\Xi_{1 i}$ is defined in (11).
Note that

$$
\begin{aligned}
\int_{t-h_{U}}^{t} \alpha(t, s) d s=\int_{t-h_{U}}^{t}\left[\frac{\dot{x}(s)}{\int_{s}^{t} \dot{x}(u) d u}\right] d s \\
=\left[\frac{\int_{t-h_{U}}^{t} \dot{x}(s) d s}{\int_{t-h_{U}}^{t} x(s) d s} \int_{t-h_{U}}^{t} \dot{x}(u) d u\right] \\
=\left[\frac{\int_{t-h(t)}^{t} x(s) d s+\int_{t-h_{U}}^{t-h(t)} x(s) d s}{\int_{t-h(t)}^{t} x(s) d s}\right] \\
=\left[\frac{\left.e_{1}-e_{3}, e_{6}+e_{7}, h_{U} e_{1}-e_{6}-e_{7}\right]^{T} \zeta(t) .}{h_{U} x(t)-\int_{t-h(t)}^{t} x(s) d s-\int_{U}}\right]
\end{aligned}
$$

From (17), calculation of $\mathscr{L} V_{2}\left(x_{t}, i\right)$ leads to

$$
\begin{aligned}
& \mathscr{L} V_{2}\left(x_{t}, i\right)=\alpha^{T}(t, t) \mathcal{N}_{i} \alpha(t, t) \cdot \frac{d}{d t}(t)-\alpha^{T}(t, t \\
& \left.-h_{U}\right) \mathcal{N}_{i} \alpha\left(t, t-h_{U}\right)^{T} \cdot \frac{d}{d t}\left(t-h_{U}\right) \\
& +\int_{t-h_{U}}^{t} \underbrace{\frac{d}{d t}\left(\alpha^{T}(t, s) \mathcal{N}_{i} \alpha(t, s)\right)}_{2 \alpha^{T}(t, s) \mathcal{N}_{i}(d \alpha(t, s) / d t)} d s+\int_{t-h_{U}}^{t} \alpha^{T}(t, s) \\
& \cdot\left(\sum_{j=1}^{s} \pi_{i j} \mathcal{N}_{j}\right) \alpha(t, s) d s=\zeta^{T}(t)\left\{\left[e_{4}, e_{1}, 0_{9 n \cdot n}\right]\right. \\
& \cdot \mathcal{N}_{i}\left[e_{4}, e_{1}, 0_{9 n \cdot n}\right]^{T}-\left[e_{5}, e_{3}, e_{1}-e_{3}\right] \mathcal{N}_{i}\left[e_{5}, e_{3}, e_{1}\right. \\
& \left.-e_{3}\right]^{T}+\operatorname{Sym}\left\{\left[e_{1}-e_{3}, e_{6}+e_{7}, h_{U} e_{1}-e_{6}-e_{7}\right]\right. \\
& \left.\left.\cdot \mathcal{N}_{i}\left[0_{9 n \cdot n}, 0_{9 n \cdot n}, e_{4}\right]^{T}\right\}\right\} \zeta(t)+\int_{t-h_{U}}^{t} \alpha^{T}(t, s) \\
& \cdot\left(\sum_{j=1}^{s} \pi_{i j} \mathcal{N}_{j}\right) \alpha(t, s) d s=\zeta^{T}(t) \Xi_{2 i} \zeta(t) \\
& +\int_{t-h_{U}}^{t} \alpha^{T}(t, s)\left(\sum_{j=1}^{s} \pi_{i j} \mathcal{N}_{j}\right) \alpha(t, s) d s .
\end{aligned}
$$

An upper bound of $\mathscr{L} V_{3}\left(x_{t}, i\right)$ can be obtained as

$$
\begin{gathered}
\mathscr{L} V_{3}\left(x_{t}, i\right) \leq\left[\begin{array}{c}
x(t) \\
0_{n \cdot 1}
\end{array}\right]^{T} \mathscr{G}_{i}\left[\begin{array}{c}
x(t) \\
0_{n \cdot 1}
\end{array}\right]-\left(1-h_{D}\right) \\
\cdot\left[\begin{array}{c}
x(t-h(t)) \\
\int_{t-h(t)}^{t} \dot{x}(s) d s
\end{array}\right]^{T} \mathscr{G}_{i}\left[\begin{array}{c}
x(t-h(t)) \\
\int_{t-h(t)}^{t} \dot{x}(s) d s
\end{array}\right]
\end{gathered}
$$




$$
\begin{aligned}
& +2 \int_{t-h(t)}^{t} \beta^{T}(t, s) \mathscr{G} \frac{\partial \beta(t, s)}{\partial t} d s+\int_{t-h(t)}^{t} \beta^{T}(t, s) \\
& \cdot\left(\sum_{j=1}^{s} \pi_{i j} \mathscr{G}_{j}\right) \beta(t, s) d s=\zeta^{T}(t) \\
& \cdot\left\{\left[e_{1}, 0_{9 n \cdot n}\right] \mathscr{G}_{i}\left[e_{1}, 0_{9 n \cdot n}\right]^{T}\right. \\
& -\left(1-h_{D}\right)\left[e_{2}, e_{1}-e_{2}\right] \mathscr{G}_{i}\left[e_{2}, e_{1}-e_{2}\right]^{T} \\
& \left.+\operatorname{Sym}\left\{\left[e_{6}, h(t) e_{1}-e_{6}\right] \mathscr{G}_{i}\left[0_{9 n \cdot n}, e_{4}\right]^{T}\right\}\right\} \zeta(t) \\
& +\int_{t-h(t)}^{t} \beta^{T}(t, s)\left(\sum_{j=1}^{s} \pi_{i j} \mathscr{G}_{j}\right) \beta(t, s) d s=\zeta^{T}(t) \\
& \cdot \Xi_{3 i[h(t)]} \zeta(t) \\
& +\int_{t-h(t)}^{t} \beta^{T}(t, s)\left(\sum_{j=1}^{s} \pi_{i j} \mathscr{G}_{j}\right) \beta(t, s) d s .
\end{aligned}
$$

Inspired by the work of [32], for any symmetric matrices $P_{i} \in$ $\mathbb{R}^{n \times n}(i=1,2)$, the following two zero equalities are satisfied:

$$
\begin{aligned}
0 & =h_{U}\left\{x^{T}(t) P_{1} x(t)-x^{T}(t-h(t)) P_{1} x(t-h(t))\right. \\
& \left.-2 \int_{t-h(t)}^{t} x^{T}(s) P_{1} \dot{x}(s) d s\right\}, \\
0 & =h_{U}\left\{x^{T}(t-h(t)) P_{2} x(t-h(t))\right. \\
& -x^{T}\left(t-h_{U}\right) P_{2} x\left(t-h_{U}\right) \\
& \left.-2 \int_{t-h_{U}}^{t-h(t)} x^{T}(s) P_{2} \dot{x}(s) d s\right\} .
\end{aligned}
$$

By summing the two zero equalities in (20), we have

$$
\begin{aligned}
0= & \zeta^{T}(t) \Xi_{5} \zeta(t)-2 h_{U} \int_{t-h(t)}^{t} x^{T}(s) P_{1} \dot{x}(s) d s \\
& -2 h_{U} \int_{t-h_{U}}^{t-h(t)} x^{T}(s) P_{2} \dot{x}(s) d s .
\end{aligned}
$$

Let $\varphi(t, s)=\int_{s}^{t} \alpha^{T}(t, u) Q \alpha(t, u) d u$. By using the similar methods presented in (18) to (19), the calculation of $\mathscr{L} V_{4}\left(x_{t}, i\right)$ can be represented as

$$
\begin{aligned}
& \mathscr{L} V_{4}\left(x_{t}, i\right)=\underbrace{\left.h_{U} \varphi(t, s)\right|_{s=t}}_{0}-\left.h_{U} \varphi(t, s)\right|_{s=t-h_{U}} \\
& +h_{U} \int_{t-h_{U}}^{t} \frac{\partial \varphi(t, s)}{\partial t} d s=-\left.h_{U} \varphi(t, s)\right|_{s=t-h_{U}} \\
& +h_{U} \int_{t-h_{U}}^{t}\left\{\alpha^{T}(t, t) \mathcal{Q} \alpha(t, t)\right. \\
& \left.+2 \int_{s}^{t} \alpha^{T}(t, u) Q \frac{\partial \alpha(t, u)}{\partial t} d u\right\} d s=h_{U}^{2} \alpha^{T}(t, t) \\
& \cdot \mathcal{Q} \alpha(t, t)-h_{U} \int_{t-h_{U}}^{t} \alpha^{T}(t, s) \mathbb{Q} \alpha(t, s) d s \\
& +2 h_{U} \int_{t-h_{U}}^{t} \int_{s}^{t} \alpha^{T}(t, u) Q \frac{\partial \alpha(t, u)}{\partial t} d u d s=\zeta^{T}(t) \\
& \left\{\left[e_{4}, e_{1}, 0_{9 n \cdot n}\right]\left(h_{U}^{2} Q\right)\left[e_{4}, e_{1}, 0_{9 n \cdot n}\right]^{T}+2 h_{U}\left[h_{U} e_{1}\right.\right. \\
& \left.-e_{6}-e_{7}, e_{8}+e_{9},\left(\frac{h_{U}^{2}}{2}\right) e_{1}-e_{8}-e_{9}\right] \\
& \left.\cdot Q\left[0_{9 n \cdot n}, 0_{9 n \cdot n}, e_{4}\right]^{T}\right\} \zeta(t)-h_{U} \int_{t-h_{U}}^{t} \alpha^{T}(t, s) \\
& \cdot \mathcal{Q} \alpha(t, s) d s=\zeta^{T}(t) \Xi_{4} \zeta(t)-h_{U} \int_{t-h_{U}}^{t} \alpha^{T}(t, s) \\
& \text { - } Q \alpha(t, s) d s
\end{aligned}
$$

Here, the following equations are utilized in (22):

$$
\begin{aligned}
& 2 h_{U} \int_{t-h_{U}}^{t} \int_{s}^{t} \alpha^{T}(t, u) Q \frac{\partial \alpha(t, u)}{\partial t} d u d s=2 h_{U} \int_{t-h_{U}}^{t} \int_{s}^{t}\left[\frac{\dot{x}(u)}{\int_{u}^{t} \dot{x}(u) d u}\right]^{T} Q\left[\frac{0_{n \cdot 1}}{\frac{0_{n \cdot 1}}{\dot{x}(t)}}\right] d u d s \\
& =2 h_{U}\left[\frac{\int_{t-h_{U}}^{t} \int_{s}^{t} \dot{x}(u) d u d s}{\frac{\int_{t-h_{U}}^{t} \int_{s}^{t} x(u) d u d s}{\int_{t-h_{U}}^{t} \int_{s}^{t} \int_{u}^{t} \dot{x}(v) d v d u d s}}\right]^{T} Q\left[\begin{array}{c}
0_{n \cdot 1} \\
\hline \frac{0_{n \cdot 1}}{\dot{x}(t)}
\end{array}\right]
\end{aligned}
$$




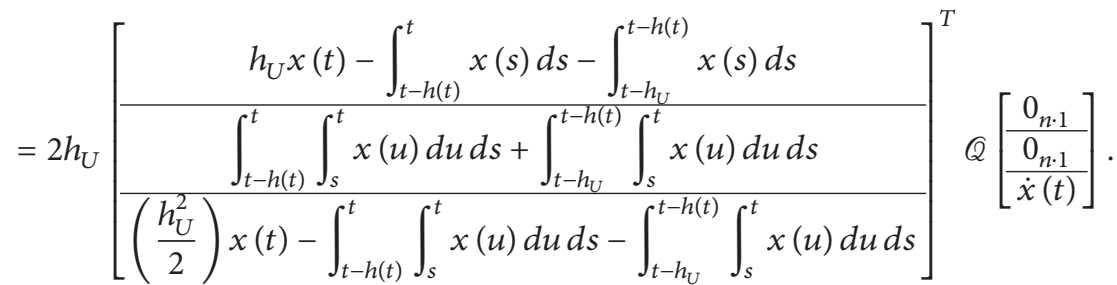

With the consideration of $\int_{t-h_{U}}^{t} \alpha^{T}(t, s)\left(\sum_{j=1}^{s} \pi_{i j} \mathcal{N}_{j}\right) \alpha(t, s) d s$ in (18), $\int_{t-h(t)}^{t} \beta^{T}(t, s)\left(\sum_{j=1}^{s} \pi_{i j} \mathscr{G}_{j}\right) \beta(t, s) d s$ in (19), and the two integral terms $-2 h_{U} \int_{t-h(t)}^{t} x^{T}(s) P_{1} \dot{x}(s) d s$ and $-2 h_{U} \int_{t-h_{U}}^{t-h(t)} x^{T}(s) P_{2} \dot{x}(s) d s$ in (21), the last integral term $-h_{U} \int_{t-h_{U}}^{t} \alpha_{2}^{T}(t, s) Q_{1} \alpha_{2}(t, s) d s$ at (22) with the addition of integral terms mentioned above can be estimated by the use of (a) in Lemma 2 and reciprocally convex optimization approach [9] as

$$
\begin{aligned}
& -h_{U} \int_{t-h_{U}}^{t} \alpha^{T}(t, s) \mathcal{Q} \alpha(t, s) d s-2 h_{U} \int_{t-h(t)}^{t} x^{T}(s) P_{1} \dot{x}(s) d s \\
& -2 h_{U} \int_{t-h_{U}}^{t-h(t)} x^{T}(s) P_{2} \dot{x}(s) d s \\
& +\int_{t-h_{U}}^{t} \alpha^{T}(t, s)\left(\sum_{j=1}^{s} \pi_{i j} \mathcal{N}_{j}\right) \alpha(t, s) d s \\
& +\int_{t-h(t)}^{t} \beta^{T}(t, s)\left(\sum_{j=1}^{s} \pi_{i j} \mathscr{G}_{j}\right) \beta(t, s) d s=-h_{U} \int_{t-h(t)}^{t} \alpha^{T}(t, s) \\
& (\underbrace{Q_{\text {aug1 }}-\left(\frac{1}{h_{U}}\right) \sum_{j=1}^{s} \pi_{i j} \mathcal{N}_{j}-\left(\frac{1}{h_{U}}\right)\left[\begin{array}{c|c}
0_{n} & 0_{n \cdot 2 n} \\
\hline 0_{2 n \cdot n} & \sum_{j=1}^{s} \pi_{i j} \mathscr{G}_{j}
\end{array}\right]}_{\Theta_{1 i}})
\end{aligned}
$$

$\alpha(t, s) d s$

$$
-h_{U} \int_{t-h_{U}}^{t-h(t)} \alpha^{T}(t, s)(\underbrace{\widehat{Q}_{\text {aug } 2}-\left(\frac{1}{h_{U}}\right) \sum_{j=1}^{s} \pi_{i j} \mathcal{N}_{j}}_{\Theta_{2 i}}) \alpha(t, s) d s,
$$

where

$$
\begin{aligned}
& \mathbb{Q}_{\text {aug1 }}=\mathbb{Q}+\left[\begin{array}{lll}
0_{n} & P_{1} & 0_{n} \\
P_{1} & 0_{n} & 0_{n} \\
0_{n} & 0_{n} & 0_{n}
\end{array}\right], \\
& \mathbb{Q}_{\text {aug2 }}=Q+\left[\begin{array}{lll}
0_{n} & P_{2} & 0_{n} \\
P_{2} & 0_{n} & 0_{n} \\
0_{n} & 0_{n} & 0_{n}
\end{array}\right],
\end{aligned}
$$

which were defined in (11).
With the use of Lemma 2, the integral term $-h_{U} \int_{t-h(t)}^{t} \alpha^{T}(t, s) \Theta_{1 i} \alpha(t, s) d s$ can be bounded as

$$
\begin{aligned}
& -h_{U} \int_{t-h(t)}^{t} \alpha^{T}(t, s) \Theta_{1 i} \alpha(t, s) d s \leq-\frac{h_{U}}{h(t)}\left(\int_{t-h(t)}^{t} \alpha(t, s) d s\right)^{T} \\
& \cdot \Theta_{1 i}\left(\int_{t-h(t)}^{t} \alpha(t, s) d s\right) \\
& -\frac{3 h_{U}}{h(t)}\left(\int_{t-h(t)}^{t} \alpha(t, s) d s-\frac{2}{h(t)} \int_{t-h(t)}^{t} \int_{s}^{t} \alpha(t, u) d u d s\right)^{T} \\
& \cdot \Theta_{1 i}\left(\int_{t-h(t)}^{t} \alpha(t, s) d s-\frac{2}{h(t)} \int_{t-h(t)}^{t} \int_{s}^{t} \alpha(t, u) d u d s\right) \\
& =-\frac{h_{U}}{h(t)}\left(\int_{t-h(t)}^{t} \alpha(t, s) d s\right)^{T} \Theta_{1 i}\left(\int_{t-h(t)}^{t} \alpha(t, s) d s\right) \\
& -\frac{3 h_{U}}{h(t)}\left(h(t) \int_{t-h(t)}^{t} \alpha(t, s) d s-2 \int_{t-h(t)}^{t} \int_{s}^{t} \alpha(t, u) d u d s\right)^{T} \\
& \cdot\left(\frac{\Theta_{1 i}}{h^{2}(t)}\right) \\
& \cdot\left(h(t) \int_{t-h(t)}^{t} \alpha(t, s) d s-2 \int_{t-h(t)}^{t} \int_{s}^{t} \alpha(t, u) d u d s\right) \\
& \leq-\frac{h_{U}}{h(t)}\left(\int_{t-h(t)}^{t} \alpha(t, s) d s\right)^{T} \Theta_{1 i}\left(\int_{t-h(t)}^{t} \alpha(t, s) d s\right) \\
& -\frac{3 h_{U}}{h(t)}\left(h(t) \int_{t-h(t)}^{t} \alpha(t, s) d s-2 \int_{t-h(t)}^{t} \int_{s}^{t} \alpha(t, u) d u d s\right)^{T} \\
& \cdot\left(\frac{\Theta_{1 i}}{h_{U}^{2}}\right) \\
& \cdot\left(h(t) \int_{t-h(t)}^{t} \alpha(t, s) d s-2 \int_{t-h(t)}^{t} \int_{s}^{t} \alpha(t, u) d u d s\right) \\
& =-\frac{h_{U}}{h(t)}\left[\frac{x(t)-x(t-h(t))}{\int_{t-h(t)}^{t} x(s) d s}\right]^{t} \\
& \cdot \Theta_{1 i}\left[\frac{x(t)-x(t-h(t))}{\int_{t-h(t)}^{t} x(s) d s}\right]
\end{aligned}
$$




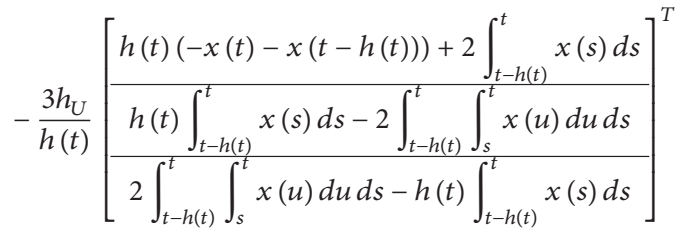

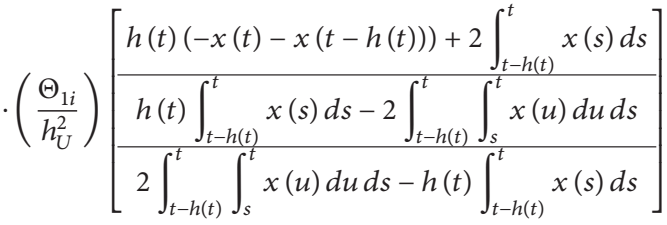

$$
\begin{aligned}
& =-\frac{h_{U}}{h(t)} \zeta^{T}(t) \Lambda_{1[h(t)]}^{T} \Omega_{1 i} \Lambda_{1[h(t)]} \zeta(t),
\end{aligned}
$$

where

$$
\begin{aligned}
& \Lambda_{1[h(t)]}=\left[e_{1}-e_{2}, e_{6}, h(t) e_{1}-e_{6}, h(t)\left(-e_{1}-e_{2}\right)\right. \\
& \left.+2 e_{6}, h(t) e_{6}-2 e_{8}, 2 e_{8}-h(t) e_{6}\right]^{T}, \\
& \Omega_{1 i}=\left[\begin{array}{cc}
\Theta_{1 i} & 0_{3 n} \\
0_{3 n} & \frac{\left(3 \Theta_{1 i}\right)}{h_{U}^{2}}
\end{array}\right] .
\end{aligned}
$$

The other integral term $-h_{U} \int_{t-h_{U}}^{t-h(t)} \alpha^{T}(t, s) \Theta_{1 i} \alpha(t, s) d s$ can be estimated as

$$
\begin{aligned}
& -h_{U} \int_{t-h_{U}}^{t-h(t)} \alpha^{T}(t, s) \Theta_{2 i} \alpha(t, s) d s \\
& \leq-\frac{h_{U}}{h_{U}-h(t)}\left(\int_{t-h_{U}}^{t-h(t)} \alpha(t, s) d s\right)^{T} \\
& \cdot \Theta_{2 i}\left(\int_{t-h_{U}}^{t-h(t)} \alpha(t, s) d s\right) \\
& -\frac{3 h_{U}}{h_{U}-h(t)}\left(\int_{t-h_{U}}^{t-h(t)} \alpha(t, s) d s\right. \\
& \left.-\frac{2}{h_{U}-h(t)} \int_{t-h_{U}}^{t-h(t)} \int_{s}^{t-h(t)} \alpha(t, u) d u d s\right)^{T} \\
& \cdot \Theta_{2 i}\left(\int_{t-h_{U}}^{t-h(t)} \alpha(t, s) d s\right. \\
& -\frac{2}{h_{U}-h(t)}\left(\left(h_{U}-h(t)\right) \int_{t-h_{U}}^{t-h(t)} \alpha(t, s) d s\right. \\
& \left.-\Theta_{2 i}\left(\int_{t-h_{U}}^{t-h(t)} \alpha(t, s) d s\right) \int_{t-h_{U}}^{t-h(t)} \alpha(t, u) d u d s\right)_{s}^{T} \\
& =-\frac{h_{U}}{h_{U}-h(t)}\left(\int_{t-h_{U}}^{t-h(t)} \alpha(t, s) d s\right)^{T}
\end{aligned}
$$

$$
\begin{aligned}
& \left.-2 \int_{t-h_{U}}^{t-h(t)} \int_{s}^{t-h(t)} \alpha(t, u) d u d s\right)^{T}\left(\frac{\Theta_{2 i}}{\left(h_{U}-h(t)\right)^{2}}\right) \\
& \cdot\left(\left(h_{U}-h(t)\right) \int_{t-h_{U}}^{t-h(t)} \alpha(t, s) d s\right. \\
& \left.-2 \int_{t-h_{U}}^{t-h(t)} \int_{s}^{t-h(t)} \alpha(t, u) d u d s\right) \\
& \leq-\frac{h_{U}}{h_{U}-h(t)}\left(\int_{t-h_{U}}^{t-h(t)} \alpha(t, s) d s\right)^{T} \\
& \cdot \Theta_{2 i}\left(\int_{t-h_{U}}^{t-h(t)} \alpha(t, s) d s\right) \\
& -\frac{3 h_{U}}{h_{U}-h(t)}\left(\left(h_{U}-h(t)\right) \int_{t-h_{U}}^{t-h(t)} \alpha(t, s) d s\right. \\
& \left.-2 \int_{t-h_{U}}^{t-h(t)} \int_{s}^{t-h(t)} \alpha(t, u) d u d s\right)^{T}\left(\frac{\Theta_{2 i}}{h_{U}^{2}}\right) \\
& \cdot\left(\left(h_{U}-h(t)\right) \int_{t-h_{U}}^{t-h(t)} \alpha(t, s) d s\right. \\
& \left.-2 \int_{t-h(t)}^{t-h(t)} \alpha(t, u) d u d s\right) .
\end{aligned}
$$

Since

$$
\begin{aligned}
& \int_{t-h_{U}}^{t-h(t)} \alpha(t, s) d s=\left[\frac{\frac{x(t-h(t))-x\left(t-h_{U}\right)}{\int_{t-h_{U}}^{t-h(t)} x(s) d s}}{\int_{t-h_{U}}^{t-h(t)} \int_{s}^{t} \dot{x}(u) d u d s}\right] \\
& =\left[e_{2}-e_{3}, e_{7},\left(h_{U}-h(t)\right) e_{1}-e_{7}\right]^{T} \zeta(t), \\
& 2 \int_{t-h_{U}}^{t-h(t)} \int_{s}^{t h(t)} \int_{u}^{t} \dot{x}(v) d v d u d s \\
& =2 \int_{t-h_{U}}^{t-h(t)} \int_{s}^{t-h(t)}(x(t)-x(u)) d u d s=2 \\
& \frac{\left(h_{U}-h(t)\right)^{2}}{2} x(t)-2 \int_{t-h_{U}}^{t-h(t)} \int_{s}^{t-h(t)} x(u) d u d s \\
& \underbrace{-2 \int_{t-h_{U}}^{t-h(t)} \int_{t-h(t)}^{t} x(u) d u d s+2 \int_{t-h_{U}}^{t-h(t)} \int_{t-h(t)}^{t} x(u) d u d s}_{=0} \\
& =\left(h_{U}-h(t)\right)^{2} x(t)-2 \int_{t-h_{U}}^{t-h(t)} \int_{s}^{t} x(u) d u d s \\
& +\underbrace{2 \int_{t-h_{U}}^{t-h(t)} \int_{t-h(t)}^{t} x(u) d u d s}_{=2 \cdot\left(h_{U}-h(t)\right) \int_{t-h(t)}^{t} x(s) d s}
\end{aligned}
$$


from (28) to (29), we have

$$
\begin{aligned}
& -h_{U} \int_{t-h_{U}}^{t-h(t)} \alpha^{T}(t, s) \Theta_{2 i} \alpha(t, s) d s \leq-\frac{h_{U}}{h_{U}-h(t)}\left[\frac{x(t-h(t))-x\left(t-h_{U}\right)}{\int_{t-h_{U}}^{t-h(t)} x(s) d s}\right]^{T} \\
& \cdot \Theta_{2 i}\left[\frac{x(t-h(t))-x\left(t-h_{U}\right)}{\int_{t-h_{U}}^{t-h(t)} x(s) d s}\right] \\
& -\frac{3 h_{U}}{h_{U}-h(t)}\left[\frac{\left(h_{U}-h(t)\right)\left(-x(t-h(t))-x\left(t-h_{U}\right)\right)+2 \int_{t-h_{U}}^{t-h(t)} x(s) d s}{\frac{\left(h_{U}-h(t)\right) \int_{t-h_{U}}^{t-h(t)} x(s) d s-2 \int_{t-h_{U}}^{t-h(t)} \int_{s}^{t} x(u) d u d s+2\left(h_{U}-h(t)\right) \int_{t-h(t)}^{t} x(s) d s}{-\left(h_{U}-h(t)\right)\left(2 \int_{t-h(t)}^{t} x(s) d s+\int_{t-h_{U}}^{t-h(t)} x(s) d s\right)+2 \int_{t-h(t)}^{t} \int_{s}^{t} x(u) d u d s}}\right]^{T}\left(\frac{\Theta_{2 i}}{h_{U}^{2}}\right)
\end{aligned}
$$

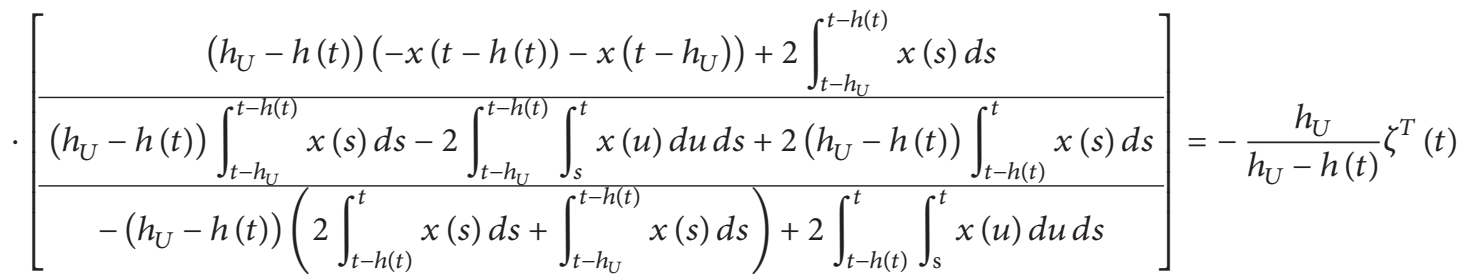

$$
\begin{aligned}
& \cdot \Lambda_{2[h(t)]}^{T} \Omega_{2 i} \Lambda_{2[h(t)]} \zeta(t),
\end{aligned}
$$

where

$$
\begin{aligned}
& \Lambda_{2[h(t)]}=\left[e_{2}-e_{3}, e_{7},\left(h_{U}-h(t)\right) e_{1}\right. \\
& -e_{7},\left(h_{U}-h(t)\right)\left(-e_{2}-e_{3}\right)+2 e_{7},\left(h_{U}-h(t)\right) e_{7} \\
& \left.-2 e_{9},-\left(h_{U}-h(t)\right)\left(2 e_{6}+e_{7}\right)+2 e_{9}\right]^{T}, \\
& \Omega_{2 i}=\left[\begin{array}{ll}
\Theta_{2 i} & 0_{3 n} \\
0_{3 n} & \frac{\left(3 \Theta_{2 i}\right)}{h_{U}^{2}}
\end{array}\right] .
\end{aligned}
$$

From (22) to (30), by utilizing reciprocally convex optimization approach [9], it can be confirmed that

$$
\begin{gathered}
\mathscr{L} V_{4}\left(x_{t}, i\right)-2 h_{U} \int_{t-h(t)}^{t} x^{T}(s) P_{1} \dot{x}(s) d s \\
-2 h_{U} \int_{t-h_{U}}^{t-h(t)} x^{T}(s) P_{2} \dot{x}(s) d s
\end{gathered}
$$

$$
\begin{aligned}
& +\int_{t-h_{U}}^{t} \alpha^{T}(t, s)\left(\sum_{j=1}^{s} \pi_{i j} \mathcal{N}_{j}\right) \alpha(t, s) d s \\
& +\int_{t-h(t)}^{t} \beta^{T}(t, s)\left(\sum_{j=1}^{s} \pi_{i j} \mathscr{G}_{j}\right) \beta(t, s) d s \\
& \leq \zeta^{T}(t)\left(\Xi_{4}-\Lambda_{3[h(t)]}^{T} \Omega_{3 i} \Lambda_{3[h(t)]}\right) \zeta(t),
\end{aligned}
$$

where $\Lambda_{3[h(t)]}=\left[\Lambda_{1[h(t)]}^{T}, \Lambda_{2[h(t)]}^{T}\right]^{T}$ and $\Omega_{3 i}=\left[\begin{array}{cc}\Omega_{1 i} & \mathcal{S} \\ \mathcal{S}^{T} & \Omega_{2 i}\end{array}\right]>0$.

From (13) to (32), an upper bound of $\mathscr{L} V\left(x_{t}, i\right)$ with the addition of (21) can be represented as

$$
\begin{aligned}
& \mathscr{L} V\left(x_{t}, i\right)+\zeta^{T}(t) \Xi_{5} \zeta(t) \\
& \quad-2 h_{U} \int_{t-h(t)}^{t} x^{T}(s) P_{1} \dot{x}(s) d s \\
& \quad-2 h_{U} \int_{t-h_{U}}^{t-h(t)} x^{T}(s) P_{2} \dot{x}(s) d s \leq \zeta^{T}(t)
\end{aligned}
$$




$$
\begin{aligned}
& \{\underbrace{\left\{\Xi_{1 i}+\Xi_{2 i}+\Xi_{3 i[h(t)]}+\Xi_{4}+\Xi_{5}\right.} \\
& \Sigma_{i[h(t)]} \\
& \left.-\Lambda_{3[h(t)]}^{T} \Omega_{3 i} \Lambda_{3[h(t)]}\right\} \zeta(t) .
\end{aligned}
$$

By utilizing Lemma 3, the following inequality

$$
\zeta^{T}(t)\left(\Sigma_{i[h(t)]}-\Lambda_{3[h(t)]}^{T} \Omega_{3 i} \Lambda_{3[h(t)]}\right) \zeta(t)<0
$$

subject to $0=\Upsilon_{i} \zeta(t)$ is equivalent to

$$
\left(\Upsilon_{i}^{\perp}\right)^{T}\left(\Sigma_{i[h(t)]}-\Lambda_{3[h(t)]}^{T} \Omega_{3 i} \Lambda_{3[h(t)]}\right) \Upsilon_{i}^{\perp}<0 .
$$

By Lemma 4, condition (35) can be casted into the following inequality with an appropriate dimension $\Psi$ :

$$
\begin{aligned}
& {\left[\begin{array}{l|l|l}
\left(\Upsilon_{i}^{\perp}\right)^{T}\left(\Sigma_{i[h(t)]}\right) \Upsilon_{i}^{\perp}+\operatorname{Sym}\left\{\left(\Upsilon_{i}^{\perp}\right)^{T}\left(\Lambda_{3[h(t)]}\right)^{T} \Psi\right\} & \Psi^{T} \\
\hline \Psi & -\Omega_{3 i}
\end{array}\right]} \\
& \quad<0 .
\end{aligned}
$$

It should be noted that inequality (36) is affinely dependent on $h(t)$. Therefore, if inequalities (12) hold for $k=1,2$, then inequality (36) is satisfied for $0 \leq h(t) \leq h_{U}$. Furthermore, one can see that $\Omega_{3 i}>0$ holds if inequalities (12) are satisfied. Therefore, if condition (12) holds, then there exists a sufficiently small positive scalar $\varepsilon$ such that $\mathscr{L} V\left(x_{t}, i\right)<$ $-\varepsilon\|x(t)\|^{2}$. Thus, by using the similar method in [33] and Definition 1 , system (1) is stochastically stable. This completes our proof.

In many cases, the information about an upper bound of $\dot{h}(t)$ is unknown. For this case, based on the result of Theorem 5, the corresponding stability condition will be presented in Corollary 6. In Corollary 6, for simplicity of matrix notations, some of vectors and matrices are redefined as

$$
\begin{aligned}
\widetilde{\Theta}_{1 i}= & Q_{\text {augl }}-\left(\frac{1}{h_{U}}\right) \sum_{j=1}^{s} \pi_{i j} \mathcal{N}_{j}, \\
\widetilde{\Omega}_{1 i}= & {\left[\begin{array}{cc}
\widetilde{\Theta}_{1 i} & 0_{3 n} \\
0_{3 n} & \frac{\left(3 \widetilde{\Theta}_{1 i}\right)}{h_{U}^{2}}
\end{array}\right], } \\
\widetilde{\Omega}_{3 i}= & {\left[\begin{array}{cc}
\widetilde{\Omega}_{1 i} & \mathcal{S} \\
\mathcal{S}^{T} & \Omega_{2 i}
\end{array}\right], } \\
\widetilde{\Sigma}_{i[h(t)]}= & \Xi_{1 i}+\Xi_{2 i}+\Xi_{4}+\Xi_{5}, \\
\widetilde{\Phi}_{i[h(t)]=} & \left(\Upsilon_{i}^{\perp}\right)^{T}\left(\widetilde{\Sigma}_{i[h(t)]}\right)\left(\Upsilon_{i}^{\perp}\right) \\
& +\operatorname{Sym}\left\{\left(\Upsilon_{i}^{\perp}\right)^{T}\left(\Lambda_{3 i[h(t)]}\right)^{T} \Psi\right\} .
\end{aligned}
$$

Except the above notations, all the notations defined in (11) will be used in Corollary 6 . Now, the following result is given by Corollary 6.

Corollary 6. For a given scalar $h_{U}>0$, system (1) is stochastically stable for $0 \leq h(t) \leq h_{U}$ if there exist positive definite matrices $\mathscr{R}_{i} \in \mathbb{R}^{4 n \times 4 n}, \mathcal{N}_{i} \in \mathbb{R}^{3 n \times 3 n}$, and $Q \in \mathbb{R}^{3 n \times 3 n}$, any matrices $\mathcal{S} \in \mathbb{R}^{6 n \times 6 n}$ and $\Psi \in \mathbb{R}^{12 n \times 8 n}$, and any symmetric matrices $P_{1} \in \mathbb{R}^{n \times n}$ and $P_{2} \in \mathbb{R}^{n \times n}$ satisfying the following LMIs for all $r(t)=i, i \in \mathcal{S}$ :

$$
\left[\begin{array}{cc}
\widetilde{\Phi}_{i, k} & \Psi^{T} \\
\Psi & -\widetilde{\Omega}_{3 i}
\end{array}\right]<0, \quad k=1,2,
$$

where $\left\{\widetilde{\Phi}_{i, k}\right\}_{k=1}^{2}$ means the two vertices of $\widetilde{\Phi}_{i[h(t)]}$ with the bounds of $0 \leq h(t) \leq h_{M}$. That is, $\widetilde{\Phi}_{i, 1}=\widetilde{\Phi}_{i[h(t)=0]}$ and $\widetilde{\Phi}_{i, 2}=\widetilde{\Phi}_{i\left[h(t)=h_{U}\right]}$.

Proof. Let us choose LKF as

$$
\begin{aligned}
V\left(x_{t}, i\right)= & \eta^{T}(t) \mathscr{R}_{i} \eta(t)+\int_{t-h_{U}}^{t} \alpha^{T}(t, s) \mathcal{N}_{i} \alpha(t, s) d s \\
& +\int_{t-h_{U}}^{t} \int_{s}^{t} \alpha^{T}(t, u) Q \alpha(t, u) d u d s .
\end{aligned}
$$

The proof of Corollary 6 is very similar to the proof of Theorem 5. Thus, it is omitted. This completes our proof.

Remark 7. Theorem 5 and Corollary 6 are derived based on the result of [25]. LKFs $V_{5}\left(x_{t}, i\right), V_{6}\left(x_{t}, i\right)$ of Theorem 1 in [25] are not included in this paper. Instead, an upper bound of $\mathscr{L} V_{4}\left(x_{t}, i\right)$ is derived by utilizing Wirtinger-based integral inequality.

Remark 8. Unlike the previous results [12-14], the integral terms $\int_{t-h(t)}^{t} x(s) d s, \int_{t-h_{U}}^{t-h(t)} x(s) d s, \int_{t-h(t)}^{t} \int_{s}^{t} x(u) d u d s$, and $\int_{t-h_{U}}^{t-h(t)} \int_{s}^{t} x(u) d u d s$ which were utilized as elements of augmented vector $\zeta(t)$ are not multiplied by $1 / h(t)$ or $1 /\left(h_{U}-h(t)\right)$. As shown in [10], the appearance of the terms $(1 / h(t)) \int_{t-h(t)}^{t} x(s) d s$ and $\left(1 /\left(h_{U}-h(t)\right)\right) \int_{t-h(t)}^{t} x(s) d s$ is unavoidable in utilizing Wirtinger-based integral inequality. However, with the terms $(1 / h(t)) \int_{t-h(t)}^{t} x(s) d s$ and $\left(1 /\left(h_{U}-\right.\right.$ $h(t))) \int_{t-h_{U}}^{t-h(t)} x(s) d s$ as elements of augmented vector, the derivation of $\mathscr{L}\left\{\eta^{T}(t) \mathscr{R}_{i} \eta(t)\right\}+\mathscr{L}\left\{\int_{t-h_{U}}^{t} \alpha^{T}(t, s) \mathcal{N}_{i} \alpha(t, s) d s\right\}$ is more difficult than the case of the terms $\int_{t-h(t)}^{t} x(s) d s$ and $\int_{t-h(t)}^{t} x(s) d s$ as elements of augmented vector. In this paper, with the process shown in (26), the utilized integral terms in augmented vector are not multiplied by $1 / h(t)$ or $1 /\left(h_{U}-\right.$ $h(t))$.

\section{Numerical Examples}

In this section, three numerical examples are introduced to show the improvements of the proposed methods. In the 
TABLE 1: Maximum delay bounds $h_{U}$ with $h_{D}=0$ and various $\pi_{11}$ (Example 1).

\begin{tabular}{lcccc}
\hline Methods & $\pi_{11}=-0.1$ & $\pi_{11}=-0.5$ & $\pi_{11}=-0.8$ & $\pi_{11}=-1$ \\
\hline$[18]$ & 0.6797 & 0.5794 & 0.5562 & 0.5465 \\
{$[19](m=5)$} & 0.8232 & 0.7327 & 0.7039 & 0.6934 \\
{$[22](m=2)$} & 1.2550 & 0.8816 & 0.8065 & 0.7783 \\
{$[25]$} & 1.2132 & 0.9797 & 0.9345 & 0.8986 \\
Theorem 5 & 1.3954 & 1.1138 & 1.0566 & 1.0367 \\
\hline
\end{tabular}

${ }^{*} m$ is delay-partitioning number.

examples, MATLAB, YALMIP, and SeDuMi 1.3 are used to solve LMI problems.

Example 1. Consider Markovian jump system (1) with the parameters

$$
\begin{aligned}
A_{1} & =\left[\begin{array}{cc}
-3.4888 & 0.8057 \\
-0.6451 & -3.2684
\end{array}\right], \\
A_{2} & =\left[\begin{array}{cc}
-2.4898 & 0.2895 \\
1.3396 & -0.0211
\end{array}\right], \\
A_{d 1} & =\left[\begin{array}{ll}
-0.8620 & -1.2919 \\
-0.6841 & -2.0729
\end{array}\right], \\
A_{d 2} & =\left[\begin{array}{ll}
-2.8306 & 0.4978 \\
-0.8436 & -1.0115
\end{array}\right] .
\end{aligned}
$$

In Table 1 , when $\pi_{22}=-0.8$ and $h_{D}=0$, the obtained maximum delay bounds by Theorem 5 are compared with some recent results and [25] under some various $\pi_{11}$. From Table 1, one can see that Theorem 5 significantly improves the feasible region of stability, which shows the advantages of the proposed Theorem 5.

Example 2. Consider Markovian jump system (1) where

$$
\begin{aligned}
& A_{1}=\left[\begin{array}{cc}
-2.3 & 0.8 \\
1.0 & -2.9
\end{array}\right], \\
& A_{2}=\left[\begin{array}{cc}
-1.9 & 0.2 \\
0.6 & -0.8
\end{array}\right], \\
& A_{d 1}=\left[\begin{array}{cc}
0.8 & 1.2 \\
0.7 & -3.5
\end{array}\right], \\
& A_{d 2}=\left[\begin{array}{cc}
-1.3 & -2.6 \\
0.5 & -1.4
\end{array}\right] .
\end{aligned}
$$

When $h_{D}$ is unknown and $\pi_{22}=-0.8$, in Table 2 , maximum delay bounds obtained by Corollary 6 are compared with those of $[18,21,25]$. Table 2 shows the less conservatism of Corollary 6.
TABLE 2: Maximum delay bounds $h_{U}$ with unknown $h_{D}$ and various $\pi_{11}$ (Example 2).

\begin{tabular}{lcccc}
\hline Methods & $\pi_{11}=-0.1$ & $\pi_{11}=-0.5$ & $\pi_{11}=-0.8$ & $\pi_{11}=-1$ \\
\hline$[18]$ & 0.271 & 0.271 & 0.271 & 0.271 \\
{$[21]$} & 0.500 & 0.496 & 0.493 & 0.492 \\
{$[25]$} & 0.6003 & 0.5909 & 0.5862 & 0.5836 \\
Corollary 6 & 0.6209 & 0.6166 & 0.6152 & 0.6146 \\
\hline
\end{tabular}

TABLE 3: Maximum delay bounds $h_{U}$ with $h_{D}=0.9$ and various $\pi_{11}$ (Example 3).

\begin{tabular}{lcccc}
\hline Methods & $\pi_{11}=-0.1$ & $\pi_{11}=-0.5$ & $\pi_{11}=-0.8$ & $\pi_{11}=-1$ \\
\hline$[18]$ & 1.0224 & 1.0148 & 1.0141 & 1.0130 \\
{$[26]$} & 1.3671 & 1.3565 & 1.3541 & 1.3535 \\
{$[25]$} & 1.7858 & 1.7006 & 1.6803 & 1.6713 \\
Theorem 5 & 1.8270 & 1.7320 & 1.7093 & 1.6999 \\
\hline
\end{tabular}

Example 3. Consider Markovian jump system (1) with the parameters

$$
\begin{aligned}
& A_{1}=\left[\begin{array}{cc}
-2 & 0 \\
0 & -0.9
\end{array}\right], \\
& A_{2}=\left[\begin{array}{cc}
-1 & 0 \\
-1 & -1
\end{array}\right], \\
& A_{d 1}=\left[\begin{array}{cc}
-1 & 0.5 \\
0.1 & -1
\end{array}\right], \\
& A_{d 2}=\left[\begin{array}{cc}
-1 & 0 \\
0.1 & -1
\end{array}\right] .
\end{aligned}
$$

In Table 3 , the results of maximum delay bounds $h_{U}$ obtained by Theorem 5 with $h_{D}=0.9, \pi_{22}=-0.8$, and various $\pi_{11}$ are listed and some recent results $[18,25,26]$ are also listed. The results in Table 3 also show that Theorem 5 provides larger delay bound than those of very recent results such as [26].

Example 4. Consider Markovian jump system (1) with the parameters

$$
\begin{aligned}
& A_{1}=\left[\begin{array}{cc}
-3.5 & 0.8 \\
-0.6 & -3.3
\end{array}\right], \\
& A_{2}=\left[\begin{array}{cc}
-2.5 & 0.3 \\
1.4 & -0.1
\end{array}\right], \\
& A_{d 1}=\left[\begin{array}{cc}
-0.9 & -1.3 \\
-0.7 & -2.1
\end{array}\right], \\
& A_{d 2}=\left[\begin{array}{cc}
-2.8 & 0.5 \\
-0.8 & -1.0
\end{array}\right] .
\end{aligned}
$$

In Table 4 , when $h_{D}=0$ and $\pi_{22}=-0.8$, maximum delay bounds $h_{U}$ obtained by Theorem 5 are listed and compared 
TABLE 4: Maximum delay bounds $h_{U}$ with $h_{D}=0$ and various $\pi_{11}$ (Example 4).

\begin{tabular}{|c|c|c|c|c|c|}
\hline Methods & $\pi_{11}=-0.4$ & $\pi_{11}=-0.55$ & $\pi_{11}=-0.7$ & $\pi_{11}=-0.85$ & $\pi_{11}=-1.00$ \\
\hline [20] & 0.6708 & 0.5894 & 0.5768 & 0.5675 & 0.5603 \\
\hline [23] & 0.6322 & 0.6120 & 0.5981 & 0.5881 & 0.5805 \\
\hline [25] & 1.0328 & 0.9933 & 0.9681 & 0.9523 & 0.9429 \\
\hline Theorem 5 & 1.1826 & 1.1335 & 1.1016 & 1.0799 & 1.0650 \\
\hline
\end{tabular}

with those of $[20,23,26]$ for various $\pi_{11}$. From the result of Table 4, the superiority of Theorem 5 can be verified.

\section{Conclusion}

In this paper, further improved results on stability for Markovian jump systems with time-varying delays were proposed in Theorem 5 and Corollary 6. With simple LKFs comparing with [25], it was shown that from three numerical examples, all the results obtained by Theorem 5 and Corollary 6 are larger than those of [25] by applying Wirtinger-based integral inequality and some new techniques to $\mathscr{L} V_{4}\left(x_{t}, i\right)$. With the ideas proposed in this paper, stability and stabilization for various systems such as multiagent systems, complex networks, and neural networks will be conducted in future works.

\section{Conflict of Interests}

The authors declare that there is no conflict of interests regarding the publication of this paper.

\section{Acknowledgments}

This research was supported by the Basic Science Research Program through the National Research Foundation of Korea (NRF) funded by the Ministry of Education, Science and Technology (2011-0009273). This work was also supported by the Human Resources Development of the Korea Institute of Energy Technology Evaluation and Planning (KETEP) grant funded by the Korea government Ministry of Trade, Industry \& Energy (no. 20144030200450).

\section{References}

[1] J.-P. Richard, "Time-delay systems: an overview of some recent advances and open problems," Automatica, vol. 39, no. 10, pp. 1667-1694, 2003.

[2] S. Xu and J. Lam, "A survey of linear matrix inequality techniques in stability analysis of delay systems," International Journal of Systems Science. Principles and Applications of Systems and Integration, vol. 39, no. 12, pp. 1095-1113, 2008.

[3] J. Sun, G. P. Liu, and J. Chen, "Delay-dependent stability and stabilization of neutral time-delay systems," International Journal of Robust and Nonlinear Control, vol. 19, no. 12, pp. 13641375, 2009.

[4] W. Qian, S. Cong, T. Li, and S. Fei, "Improved stability conditions for systems with interval time-varying delay," International Journal of Control, Automation and Systems, vol. 10, no. 6, pp. 1146-1152, 2012.
[5] O.-M. Kwon, M.-J. Park, S.-M. Lee, J. H. Park, and E.-J. Cha, "Stability for neural networks with time-varying delays via some new approaches," IEEE Transactions on Neural Networks and Learning Systems, vol. 24, no. 2, pp. 181-193, 2013.

[6] R. Rakkiyappan, Q. Zhu, and A. Chandrasekar, "Stability of stochastic neural networks of neutral type with Markovian jumping parameters: a delay-fractioning approach," Journal of the Franklin Institute, vol. 351, no. 3, pp. 1553-1570, 2014.

[7] K. Gu, "A further refinement of discretized Lyapunov functional method for the stability of time-delay systems," International Journal of Control, vol. 74, no. 10, pp. 967-976, 2001.

[8] Y. He, M. Wu, J.-H. She, and G.-P. Liu, "Delay-dependent robust stability criteria for uncertain neutral systems with mixed delays," Systems and Control Letters, vol. 51, no. 1, pp. 57-65, 2004.

[9] P. Park, J. W. Ko, and C. Jeong, "Reciprocally convex approach to stability of systems with time-varying delays," Automatica, vol. 47, no. 1, pp. 235-238, 2011.

[10] A. Seuret and F. Gouaisbaut, "Wirtinger-based integral inequality: application to time-delay systems," Automatica, vol. 49, no. 9, pp. 2860-2866, 2013.

[11] J. Cheng and L. Xiong, "Improved integral inequality approach on stabilization for continuous-time systems with time-varying input delay," Neurocomputing, vol. 160, pp. 274-280, 2015.

[12] O. M. Kwon, M. J. Park, J. H. Park, S. M. Lee, and E. J. Cha, "Analysis on passivity for uncertain neural networks with timevarying delays," Mathematical Problems in Engineering, vol. 2014, Article ID 602828, 10 pages, 2014.

[13] O. M. Kwon, M. J. Park, J. H. Park, S. M. Lee, and E. J. Cha, "On less conservative stability criteria for neural networks with timevarying delays utilizing Wirtinger-based integral inequality," Mathematical Problems in Engineering, vol. 2014, Article ID 859736, 13 pages, 2014.

[14] O. M. Kwon, M. J. Park, J. H. Park, S. M. Lee, and E. J. Cha, "Improved results on stability of linear systems with timevarying delays via Wirtinger-based integral inequality," Journal of the Franklin Institute. Engineering and Applied Mathematics, vol. 351, no. 12, pp. 5386-5398, 2014.

[15] X. Feng, K. A. Loparo, Y. Ji, and H. J. Chizeck, "Stochastic stability properties of jump linear systems," IEEE Transactions on Automatic Control, vol. 37, no. 1, pp. 38-53, 1992.

[16] X.-D. Zhao and Q.-S. Zeng, "Delay-dependent stability analysis for Markovian jump systems with interval time-varying-delays," International Journal of Automation and Computing, vol. 7, no. 2, pp. 224-229, 2010.

[17] P. Shi and F. Li, "A survey on Markovian jump systems: modeling and design," International Journal of Control, Automation and Systems, vol. 13, no. 1, pp. 1-16, 2015.

[18] S. Xu, J. Lam, and X. Mao, "Delay-dependent $H_{\infty}$ control and filtering for uncertain Markovian jump systems with timevarying delays," IEEE Transactions on Circuits and Systems. I. Regular Papers, vol. 54, no. 9, pp. 2070-2077, 2007. 
[19] Z. Fei, H. Gao, and P. Shi, "New results on stabilization of Markovian jump systems with time delay," Automatica, vol. 45, no. 10, pp. 2300-2306, 2009.

[20] Z. Wu, H. Su, and J. Chu, " $H_{\infty}$ filtering for singular Markovian jump systems with time delay," International Journal of Robust and Nonlinear Control, vol. 20, no. 8, pp. 939-957, 2010.

[21] Z. Li, Z. Fei, and H. Gao, "Stability and stabilisation of Markovian jump systems with time-varying delay: an input-output approach," IET Control Theory and Applications, vol. 6, no. 17, pp. 2601-2610, 2012.

[22] H. Huang, G. Feng, and X. Chen, "Stability and stabilization of Markovian jump systems with time delay via new Lyapunov functionals," IEEE Transactions on Circuits and Systems I: Regular Papers, vol. 59, no. 10, pp. 2413-2421, 2012.

[23] J. Wang, H. Wang, A. Xue, and R. Lu, "Delay-dependent $H_{\infty}$ control for singular Markovian jump systems with time delay," Nonlinear Analysis: Hybrid Systems, vol. 8, pp. 1-12, 2013.

[24] Q. Zhu, "Stabilization of stochastically singular nonlinear jump systems with unknown parameters and continuously distributed delays," International Journal of Control, Automation and Systems, vol. 11, no. 4, pp. 683-691, 2013.

[25] O. M. Kwon, M. J. Park, J. H. Park, S. M. Lee, and E. J. Cha, "Stability and $H_{\infty}$ performance analysis for Markovian jump systems with time-varying delays," Journal of the Franklin Institute. Engineering and Applied Mathematics, vol. 351, no. 10, pp. 4724-4748, 2014.

[26] J. Qiu, Y. Wei, and H. R. Karimi, "New approach to delaydependent $H_{\infty}$ control for continuoustime Markovian jump systems with time-varying delay and deficient transition descriptions," Journal of the Franklin Institute. Engineering and Applied Mathematics, vol. 352, no. 1, pp. 189-215, 2015.

[27] J. Cheng, H. Zhu, S. Zhong, Q. Zhong, and Y. Zeng, "Finitetime $H_{\infty}$ estimation for discretetime Markov jump systems with time-varying transition probabilities subject to average dwell time switching," Communications in Nonlinear Science and Numerical Simulation, vol. 20, no. 2, pp. 571-582, 2015.

[28] Z. Shu, J. Lam, and S. Xu, "Robust stabilization of Markovian delay systems with delay-dependent exponential estimates," Automatica, vol. 42, no. 11, pp. 2001-2008, 2006.

[29] E. K. Boukas and Z. K. Liu, Deterministic and Stochastic Time Delay Systems, Birkhäuser, Boston, Mass, USA, 2002.

[30] R. E. Skelton, T. Iwasaki, and K. M. Grigoradis, A Unified Algebraic Approach to Linear Control Design, Taylor and Francis, New York, NY, USA, 1997.

[31] C. Briat, "Convergence and equivalence results for the Jensen's inequality-application to time-delay and sampled-data systems," IEEE Transactions on Automatic Control, vol. 56, no. 7, pp. 1660-1665, 2011.

[32] S. H. Kim, P. Park, and C. Jeong, "Robust $H_{\infty}$ stabilisation of networked control systems with packet analyser," IET Control Theory and Applications, vol. 4, no. 9, pp. 1828-1837, 2010.

[33] M. J. Park, O. M. Kwon, J. H. Park, and S. M. Lee, "Simplified stability criteria for fuzzy Markovian jumping Hopfield neural networks of neutral type with interval time-varying delays," Expert Systems with Applications, vol. 39, no. 5, pp. 5625-5633, 2012. 


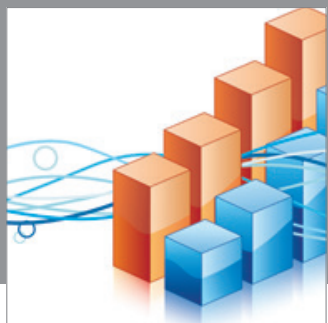

Advances in

Operations Research

mansans

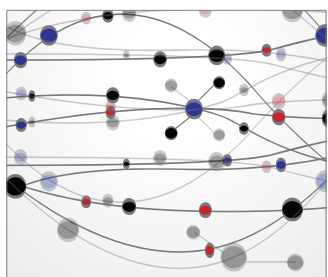

The Scientific World Journal
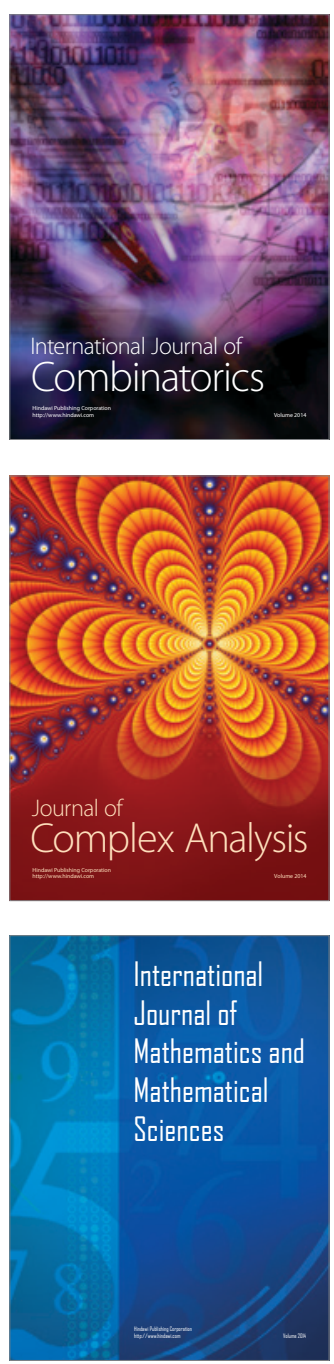
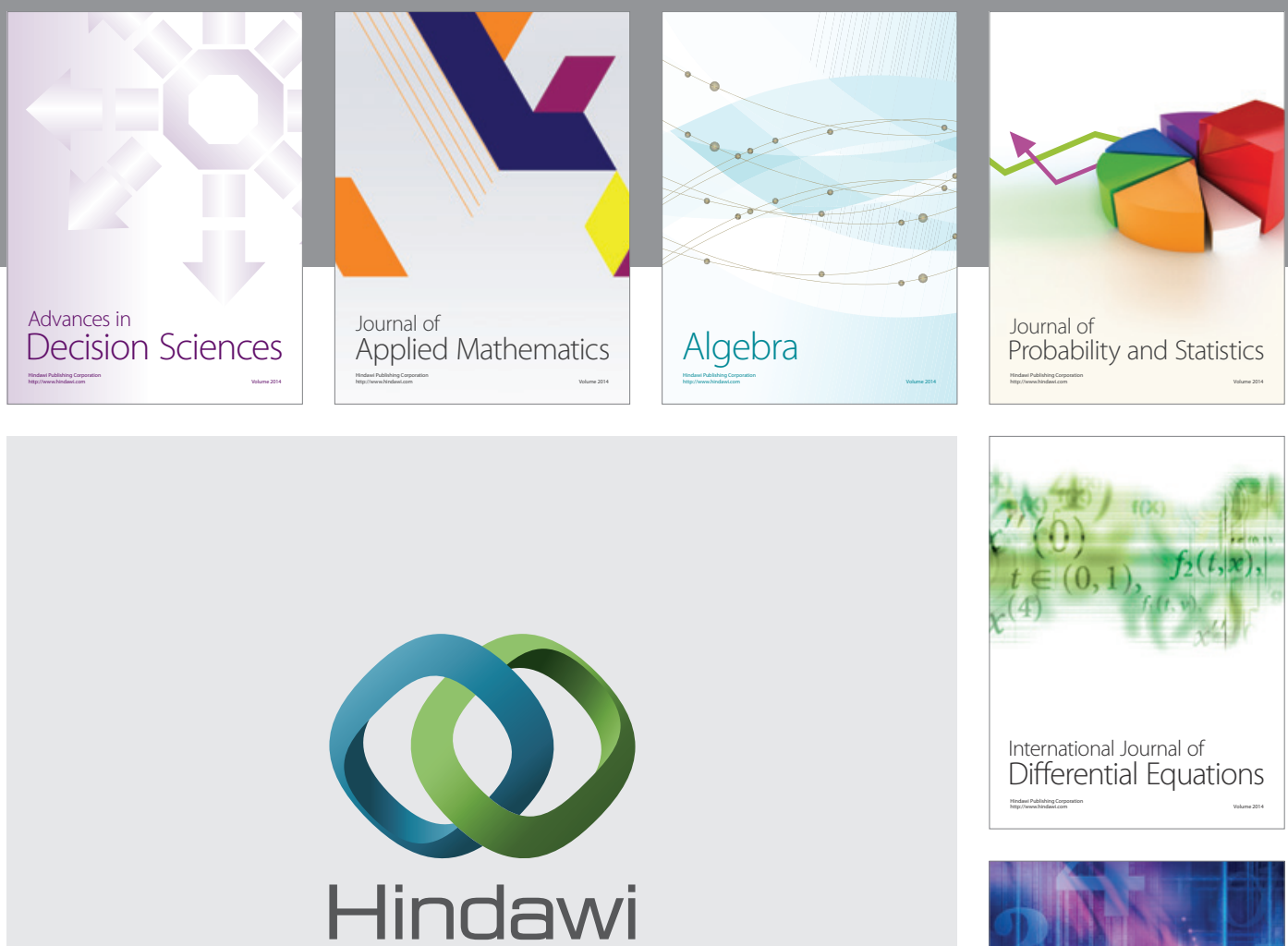

Submit your manuscripts at http://www.hindawi.com
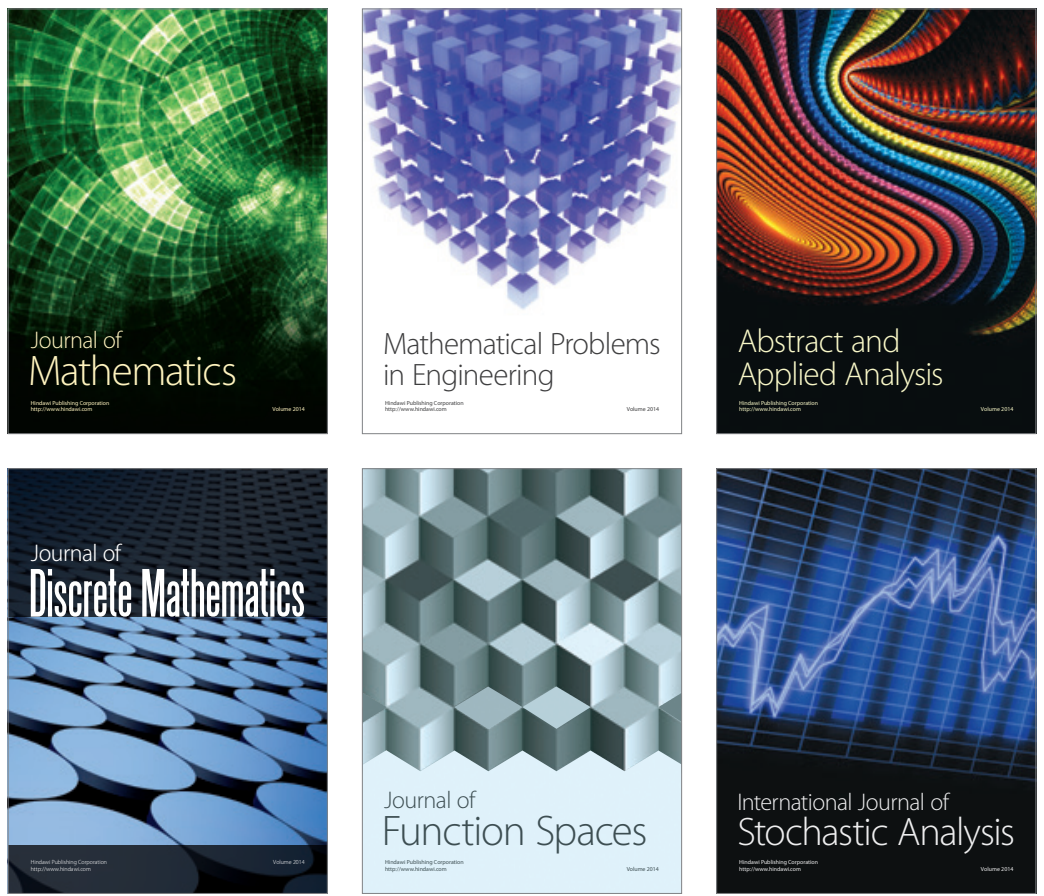

Journal of

Function Spaces

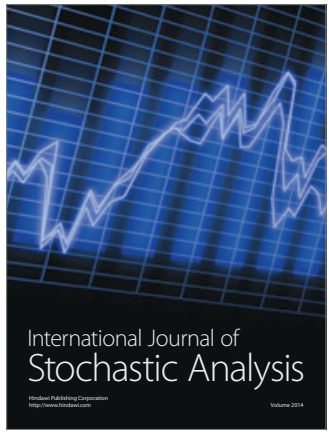

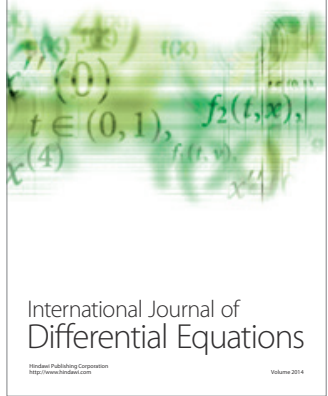
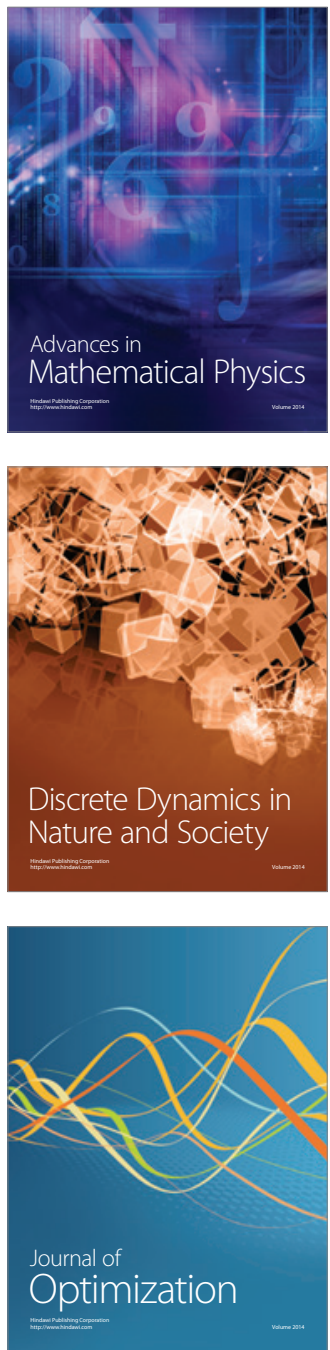